\title{
ARC-DISJOINT HAMILTONIAN CYCLES IN ROUND DECOMPOSABLE LOCALLY SEMICOMPLETE DIGRAPHS
}

\author{
RuiJuan LI ${ }^{1}$ AnD Tingting Han \\ School of Mathematical Sciences \\ Shanxi University \\ 030006 Taiyuan, P.R. China \\ e-mail: ruijuanli@sxu.edu.cn \\ tingtinghan19@163.com
}

\begin{abstract}
Let $D=(V, A)$ be a digraph; if there is at least one arc between every pair of distinct vertices of $D$, then $D$ is a semicomplete digraph. A digraph $D$ is locally semicomplete if for every vertex $x$, the out-neighbours of $x$ induce a semicomplete digraph and the in-neighbours of $x$ induce a semicomplete digraph. A locally semicomplete digraph without 2-cycle is a local tournament. In 2012, Bang-Jensen and Huang [J. Combin Theory Ser. B 102 (2012) 701-714] concluded that every 2-arc-strong locally semicomplete digraph which is not the second power of an even cycle has two arc-disjoint strong spanning subdigraphs, and proposed the conjecture that every 3strong local tournament has two arc-disjoint Hamiltonian cycles. According to Bang-Jensen, Guo, Gutin and Volkmann, locally semicomplete digraphs have three subclasses: the round decomposable; the non-round decomposable which are not semicomplete; the non-round decomposable which are semicomplete. In this paper, we prove that every 3 -strong round decomposable locally semicomplete digraph has two arc-disjoint Hamiltonian cycles, which implies that the conjecture holds for the round decomposable local tournaments. Also, we characterize the 2-strong round decomposable local tournaments each of which contains a Hamiltonian path $P$ and a Hamiltonian cycle arc-disjoint from $P$.
\end{abstract}

Keywords: locally semicomplete digraph, local tournament, round decomposable, arc-disjoint, Hamiltonian cycle, Hamiltonian path.

2010 Mathematics Subject Classification: 05C20.

\footnotetext{
${ }^{1}$ Research is supported partially by NNSFC under no. 11401353, NSF of Shanxi Province, China under no. 2016011005 and TYAL of Shanxi.
} 


\section{REFERENCES}

[1] J. Bang-Jensen and G. Gutin, Digraphs: Theory, Algorithms and Applications (Springer Monographs in Mathematics, Spring-Verlag, London, 2009). doi:10.1007/978-1-84800-998-1

[2] J. Bang-Jensen, Locally semicomplete digraph: A generalization of tournaments, J. Graph Theory 14 (1990) 371-390. doi:10.1002/jgt.3190140310

[3] J. Bang-Jensen, Y. Guo, G. Gutin and L. Volkmann, A classification of locally semicomplete digraphs, Discrete Math. 167-168 (1997) 101-114. doi:10.1016/S0012-365X(96)00219-1

[4] J. Bang-Jensen and J. Huang, Decomposing locally semicomplete digraphs into strong spanning subdigraphs, J. Combin. Theory Ser. B 102 (2012) 701-714. doi:10.1016/j.jctb.2011.09.001

[5] Y. Guo, Locally Semicomplete Digraphs (Ph.D. Thesis, RWTH Aachen University, 1995).

[6] Y. Guo, Strongly Hamiltonian-connected locally semicomplet digraphs, J. Graph Theory 21 (1996) 65-73. doi:10.1002/(SICI)1097-0118(199605)22:1〈65::AID-JGT9〉3.0.CO;2-J

[7] F. Harary and L. Moser, The theory of round robin tournaments, Amer. Math. Monthly 73 (1966) 231-246. doi: $10.2307 / 2315334$

[8] J. Huang, On the structure of local tournaments, J. Combin. Theory Ser. B 63 (1995) 200-221. doi:10.1006/jctb.1995.1016

[9] S. Li, W. Meng, Y. Guo and G. Xu, A local tournament contains a vertex whose out-arc are pseudo-girth-pancyclic, J. Graph Theory 62 (2009) 346-361. doi:10.1002/jgt.20404

[10] D. Meierling, Local tournaments with the minimum number of Hamiltonian cycles or cycles of length three, Discrete Math. 310 (2010) 1940-1948. doi:10.1016/j.disc.2010.03.003

[11] C. Thomassen, Edge-disjoint Hamiltonian paths and cycles in tournaments, Proc. Lond. Math. Soc. (3) 45 (1982) 151-168.

doi:10.1112/plms/s3-45.1.151

[12] R. Wang, A. Yang and S. Wang, Kings in locally semicomplete digraphs, J. Graph Theory 63 (2010) 279-283. doi:10.1002/jgt.20426

[13] X.H. Zhang, R.J. Li and S.J. Li, H-force sets of locally semicomplete digraphs, Discrete Appl. Math. 160 (2012) 2491-2496.

doi:10.1016/j.dam.2012.06.014

Received 17 August 2016

Revised 1 November 2016

Accepted 23 December 2016 